\title{
Announcement/Chronique
}

\section{Auguste Comte aujourd'hui Colloque de Cerisy - Juillet 2001}

\author{
Colloque international \\ (les communications auront lieu en français)
}

\begin{abstract}
Le colloque, qui fera le point des divers travaux consacrés récemment à Comte, a pour but $: 1^{\circ}$ ) de rappeler la prodigieuse diffusion, aujourd'hui presque totalement oubliée, du positivisme il y a un siècle; $2^{\circ}$ ) de montrer l'actualité de cette pensée, en science comme en politique.

En philosophie des sciences, les versions plus récentes du positivisme ont tendu à faire oublier l'enseignement de Comte. Il s'agirait donc de montrer que, cent cinquante ans après sa publication, le Cours de philosophie positive nourrit encore la réflexion des épistémologues. Les questions des rapports entre les sciences, des manières d'en considérer l'histoire et de les enseigner sont encore et toujours à repenser.

En philosophie politique, la situation se présente sous un autre jour. Alors que, notamment grâce à Canguilhem, les épistémologues français avaient toujours fait une place à Comte, on en dirait difficilement autant des politologues. Après avoir, de Littré à Maurras ou à Alain, dominé la scène intellectuelle de la Troisième République, la politique positive est soudain apparue totalement obsolète. C'est là que la réévaluation est la plus urgente et sans doute aussi la plus prometteuse. Dans la crise que traversent actuellement nos sociétés, il n'est pas du tout absurde d'estimer qu'une part croissante de nos contemporains reconnaîtra à nouveau la valeur d'une pensée politique tenue à tort pour définitivement dépassée.
\end{abstract}

Le colloque est ouvert à tous. Il se tiendra du 3 au 10 juillet 2001 au : CENTRE CULTUREL INTERNATIONAL DE CERISY-LA-SALLE

$$
\text { 50210, Cerisy-la-Salle, France }
$$

Il n'y a pas de frais d'inscription; les frais de séjour, perçus par le CCIC, sont d'environ 450 francs par jour.

Pour toute information complémentaire, comme pour les inscriptions, s'adresser au CCIC :

27, rue de Boullainvilliers, 75016 Paris

téléphone fax : 0145204203

internet : www.ccic-cerisy.asso.fr

courriel : edith.heurgon@ccic-cerisy.asso.fr 